\title{
Sobre la necesidad de considerar el futuro para tomar decisiones presentes*
}

\author{
Boris Graizbord**
}

Cuando encontramos razones para elegir estamos en vías de desarrollar una teoría de la acción colectiva y del cambio institucional.

BROMLEY, 2008: 30

La toma de decisiones es el proceso por el cual se evalúan alternativas o cursos de acción para decidir sobre ellas. En general podríamos decir que al menos dos rutas son útiles para analizar un proceso de toma de decisiones: la teórica y la empírica. La primera busca explicar cómo se toman las decisiones o cómo deberían tomarse pero también cuáles son los factores que deben considerarse al tomarlas. La segunda intenta aprender de las decisiones tomadas y de sus efectos, conocer cuáles han sido los resultados y qué factores empujaron al que las tomó.

La relevancia de pensar en la distinción entre lo que es y lo que debe ser está en la necesidad cada vez más acuciante de tomar decisiones en condiciones de riesgo o incertidumbre. Lo que define una situación de incertidumbre es la posibilidad de más de un resultado y que cada posible resultado se conozca, pero no así la probabilidad de un resultado en particular. En cambio en el riesgo sí se conoce la probabilidad del resultado. ${ }^{1}$

La incertidumbre es consubstancial con el sistema natural en el que se desarrolla la vida humana en el planeta, pues no sabemos a ciencia cierta los resultados posibles de nuestra actividad, aunque podemos conocer en algunos casos las probabilidades de que ocurra un resultado a partir de alguna acción. Esto es válido para diferentes escalas. Por ejemplo, no conocemos el posible resultado de acabar con alguna especie animal o vegetal en un ecosistema pero sí las probabi-

* Una versión de este ensayo se presentó el 24 de septiembre de 2008 en el seminario Thinking of the Present by Considering the Future organizado en la Facultad de Economía de la UNAM del 24 al 26 de septiembre de 2008, durante la visita del profesor Bromley a México.

** Lead-México / El Colegio de México. Correo electrónico: <graiz@colmex.mx>.

${ }^{1}$ Al lanzar una moneda al aire la probabilidad de atinar cara o cruz es de $50 \%$, mientras que cuando en la ruleta rusa hay una bala en una de las seis recámaras de la pistola la probabilidad de atinar es de 1 en 6 . Enfrentaríamos una situación de incertidumbre si no supiéramos si el arma está o no cargada. 
lidades de que ésta se extinga si continuamos con nuestras acciones, como sería el caso de la explotación de una pesquería. Sin embargo, es incierto el efecto que esto tendría en el sistema ecológico marino. En términos de una mayor complejidad no conocemos a ciencia cierta lo que sucedería al afectar el ciclo del carbono, como lo venimos haciendo, aunque sí sabemos que determinado nivel de emisiones de gases de efecto invernadero (GEI) en la atmósfera probablemente elevará la temperatura promedio global en determinados grados centígrados, y la probabilidad de que se presenten efectos importantes, aunque diferenciados regionalmente, en el clima.

En este marco de incertidumbre y riesgo es pertinente reflexionar sobre la manera en que las sociedades deciden colectivamente sobre las acciones que emprenden. Es éste precisamente el tema de interés que caracteriza la reflexión de Daniel Bromley a lo largo de su libro (Bromley, 2006) al insistir en desarrollar instituciones que permitan de manera pragmática tomar decisiones que afectarán a la comunidad, pero que es necesario tomarlas ante situaciones de incertidumbre y ante la necesidad de enfrentar riesgos de todo tipo.

La oportunidad de conocer al profesor Bromley y leer su libro (Bromley, 2006) se la debo a Eduardo Vega, quien además de obsequiármelo me indujo con su entusiasmo a no postergar su lectura. En lo que sigue haré un recorrido de éste (citado como 2006) y me referiré ocasionalmente al texto Sufficient Reason: Volitional Pragmatism and the Purposes of the Future (citado como 2008), que leyó el profesor en ocasión de su visita a México.

En el prefacio de su libro Bromley plantea atinadamente una propuesta fundamental: "La necesidad de articular una creencia social para predicar toda acción a favor del ambiente". La esencia de la socialización -asevera Bromley- es precisamente estabilizar las creencias pues sólo así podemos definir qué es normal, natural, correcto, "cierto", "verdadero". ${ }^{2}$ Esta idea es central si creemos que para sobrevivir como especie es imperativo tomar acciones para mitigar el cambio climático, para detener la tendencia del calentamiento global y para frenar la expoliación de recursos y servicios ambientales en el planeta.

La tarea que se propone el autor en este paradigmático libro es definir cuál es la base de esos arreglos sociales, es decir, de las institu-

${ }^{2}$ En su primera clase el profesor Bromley define "verdad": "Truth is not a property of objects and events -the 'thing in itself'. Rather, truth is a property of statements about objects and events" (2008: 13). 
ciones que definen nuestro ser como criaturas sociales. ¿Cómo es que un cambio institucional modifica, altera el comportamiento de los individuos en una sociedad? ¿Por qué se decide y se realiza ese cambio en las reglas o las instituciones? Los ejemplos están ahí: los suecos decidieron cambiar el sentido de circulación de los automóviles de la izquierda a la derecha; respondiendo al llamado de Rachel Carson se prohibió el uso del DDT en Estados Unidos; se modificó la tradición ancestral de cazar zorros en los campos de Inglaterra; Robert Owen persuadió al parlamento inglés de prohibir el trabajo infantil. ¿Se trató de decisiones racionales, o bien quienes las tomaron vieron un futuro que imaginaron que podría ser mejor? En efecto -insiste Bromley- en todos estos cambios institucionales la pregunta pertinente no es si el cambio será más eficiente, o si había alguna falla del mercado que hubiese que corregir, sino ¿queremos alcanzar un futuro imaginado, o queremos seguir igual? (2006: 8).

Sin una idea del futuro, y si no se le atribuye un valor-argumenta-, no será posible que ese futuro nos lleve a actuar en el presente. "Pensar el presente considerando el futuro" para tomar decisiones no es precisamente buscar una mayor utilidad sino creer que obtendremos resultados más convincentes, más atractivos, si implementamos tal o cual decisión o política pública (2006: 14). Para Bromley, si no adoptamos una voluntad prospectiva ${ }^{3}$ que vea la forma en que el futuro debiera o pudiera darse, y traduzca el deseo humano en acción, seguiremos aceptando los arreglos institucionales existentes que son insuficientes para tomar decisiones racionales.

La premisa epistemológica que se basa en la aceptación de creencias científicas tradicionales tipo si $Y$ entonces $X$, conecta el resultado deseado $Y$ con la acción necesaria $X$ para alcanzarlo. De tal manera que prescribe lo que debe hacerse $(X)$ para alcanzar el resultado deseado $(Y)$. Pero, además, predice que $Y$ se conseguirá si una acción $X$ se lleva a cabo (2006: 15). Por otra parte, la premisa que toma en cuenta la voluntad informa acerca de lo que debe hacerse y, en un estado democrático, lo que debe hacerse emanará de los poderes legislativo y judicial y no necesariamente de decisiones que toman en cuenta por ejemplo los planteamientos de la teoría del bienestar, que señala que el resultado $Y$ es deseado si los beneficios de $Y$ exceden los costos de $X$ (de llevar a cabo la/s acción/es $X)(2006: 16)$.

3 Bromley (2006: 217) define Prospective volition como la voluntad humana en acción, mirando el futuro, decidiendo cómo el futuro debiera desdoblarse, abrirse. 
Conviene precisar su enfoque epistémico. Para ello, y después de criticar sistemáticamente y rechazar argumentativamente los enfoques inductivo y deductivo, propone un enfoque abductivo ${ }^{4}$ en el que el científico está preocupado en descubrir las razones del resultado, pero contrariamente al enfoque deductivo no está comprometido al caso o situación como explicación (Bromley, 2006: 110), por lo que la pregunta esencial del mismo sería: ¿existe razón suficiente para explicar por qué se tomó una decisión?, en contraste con la deducción que es el resultado de la aplicación de una regla (axioma) y un caso para producir un resultado (una pretensión empírica). Es decir, que mientras la deducción produce pretensiones empíricas que pudieran resultar en propuestas teóricas, la abducción comienza con la observación de circunstancias empíricas (resultados) y después invoca axiomas específicos (reglas) y supuestos o postulados aplicables (casos) para generar propuestas (hipótesis comprobables) con la intención de explicar aquellas circunstancias observadas (2006: 96).

Me parece que este dilema es central en la discusión sobre las políticas ambientales encaminadas a alcanzar y respetar los principios del desarrollo sustentable (DS). En efecto, en la explotación de recursos, por ejemplo, los costos son asumidos hoy mientras que los beneficios que se descuentan afectan en el futuro a personas que aún no han nacido. En este caso, dice el autor, el futuro se considera en términos del presente y nos convertimos en dictadores sobre los recursos que heredaremos a las futuras generaciones. En otras palabras, actuamos para nuestros intereses y no para los de nuestros hijos, nietos, etc. Tendríamos que explicar cómo puede considerarse que estas decisiones maximizan el bienestar social en el tiempo (2006: 17). La pregunta pertinente no es cómo definir la tasa de descuento sino ¿qué es lo correcto con respecto al futuro? El problema -dice Bromley- concierne a cómo la tiranía del tiempo se resuelve en términos de todos los individuos, presentes y futuros.

${ }^{4}$ El enfoque abductivo se distingue como un "razonamiento que va del efecto a la causa (o razón). Infiere la existencia de un hecho muy diferente de cualquier cosa observada, del que, de acuerdo con leyes conocidas, algo observado necesariamente resultaría. La abducción explica los efectos" (Bromley, 2006: 217). Se trata de un viejo pero descuidado proceso de fijación de creencias. Citando a Pierce dice que el proceso de la abducción comienza cuando nos encontramos ante la necesidad de una explicación frente a circunstancias y eventos particulares: "la acción del pensamiento es excitada por una irritación causada por la duda, y cesa cuando logramos alcanzar una creencia; de tal suerte que la producción de creencias es la única función del pensamiento" (Pierce, 1957: 36, cit. en Bromley, 2006: 24). 
El dilema atrajo mi atención y en un ensayo (Graizbord, 2008) insistí en la indisoluble relación recíproca que se plantea sin resolver en la definición de Brundtland de DS entre la justicia intrageneracional y la justicia intergeneracional. ${ }^{5}$

Pero ¿dónde o cómo ubicar este texto, del que todavía queda mucho por decir? Quizá en la tensión entre determinismo explicativo o empirismo lógico y un cierto relativismo de la posmodernidad. Sospecho que el pragmatismo volitivo (volitional pragmatism) de Bromley se inscribe en el simbolismo interactivo de la tradición relativista. ${ }^{6}$ Allí encontramos, por cierto, a Simmel (individualismo), a Weber (acción social) pero también, creo, a Dewey (pragmatismo filosófico), a Mead y a Blumer (interaccionismo simbólico y pragmatismo individualista). ${ }^{7}$ O bien en la confrontación del análisis de la "hoja lisa" de la sociología explicativa, por un lado, y el análisis del mundo lleno, por otro, es decir la "hoja arrugada" por un conocimiento basado en la experiencia común, las vivencias, la interpretación, lo cualitativo de la existencia frente a los números, la medición y la cuantificación. ${ }^{8}$

De otra forma más moderna, explica Maffesoli (2008: 72), se puede decir que la unidimensionalidad del pensamiento no es apta para comprender la pluridimensionalidad de lo vivido. Al respecto, y siguiendo a Maffesoli, podemos distinguir

${ }^{5}$ En un texto reciente Roemer (2008) alude a los aspectos éticos que plantea el calentamiento global y la elevación de la temperatura causada por emisiones de gases efecto invernadero que si no se atiende rápidamente y alcanza $750 \mathrm{ppm} C O 2 \mathrm{e}$, previsto para el final de este siglo si continuamos con "business as usual", terminará con la vida en el planeta tal y como la conocemos (2008: 2). Se refiere a los de justicia intertemporal e intratemporal. Y, tomando en cuenta que las emisiones de carbono son un mal público, se pregunta: ¿cómo debemos asignar los derechos de producir este mal durante el presente y en el futuro? Éste es el aspecto intertemporal mientras que el intratemporal es ¿̇cómo los derechos para emitir carbono deben distribuirse entre naciones en ésta y la próxima generación? Roemer sugiere que la solución apropiada presupone la respuesta a la primera pregunta, lo que creo coincide con el argumento de Bromley. En efecto, me parece que la única respuesta a la segunda pregunta es tomar decisiones ahora en función de tener claro el futuro que queremos imaginar.

${ }^{6}$ Me pregunto si Bromley estaría dispuesto a publicar en la American Sociological Review o sólo en la American Journal of Sociology, revistas que representaron en su momento dos visiones (Harvard frente a Chicago).

${ }^{7}$ Véase especialmente los capítulos 12 y 13 de Alexander (2000) sobre el interaccionismo simbólico y la tradición contemporánea de la teoría social pragmatista.

${ }^{8}$ En un reciente libro Cortés (Cortés et al., 2008) habla del fin de la "guerra de los paradigmas" (p. 30) y señala que no debemos mantenernos en una crítica al positivismo lógico que desde los años treinta del siglo pasado ha evolucionado hacia el post positivismo acercándose en sus postulados ontológicos y epistemológicos al constructivismo o naturalismo (p. 31, cuadro 1). 
periodos en los que una sociedad, o un conjunto de sociedades, funciona en referencia a un valor dominante. Hay otros que parecen mantener en competencia ciertos valores diferentes, contradictorios. En el primer caso, son los momentos activos de conquista los que necesitan de una ideología unificada, de un cuerpo doctrinal eficaz que privilegia intelectualmente el concepto. En el segundo se trata de periodos más lascivos, más pasivos en los que se pone el acento menos en la extensión (el hombre amo y poseedor de la naturaleza -caro a Descartes-) y más en la intensidad, en una energía tendida en este mundo [...] que induce a un achicamiento del espacio y a una profundización de las relaciones; el lugar forja lazos [...] y es posible hacer funcionar la pluralidad de valores. [Se trata de una] lucha inexplicable entre órdenes de valores en la que descansa, diría Weber, la existencia social [p. 73].

Sólo para aclarar esta situación conviene reproducir la cita a Weber del texto de Maffesoli:

Si hay algo que en nuestros días ya no ignoramos más es que una cosa puede ser santa aunque no sea bella, y más aún porque y en la medida de que no es bella [...] Finalmente, la sabiduría popular nos enseña que una cosa puede ser verdadera, aunque no lo sea y aun cuando no sea ni bella, ni santa, ni buena [...] casos elementales en la confrontación que opone a los dioses de los diferentes órdenes y de los diferentes valores (2008: 73).

Entiendo, como explica Maffesoli (2008: 76), que es el momento de la tribu, que se constituye en la proximidad a partir de la experiencia vivida... "el nosotros fusional, el reagrupamiento al cual uno se adhiere por la ideología o por necesidad de protección”.

Suena familiar: nos reunimos en el Zócalo y decimos "NO" a los cambios. Pero creo que Bromley no aceptaría fácilmente esta conclusión. Más bien creo que le preocupa precisamente cómo "hacer funcionar la pluralidad de valores". De hecho, dice, la carga más pesada para la acción conjunta es la necesidad de enfrentar múltiples expresiones en competencia (2006: 140). O, como aparece en el texto de su conferencia: "El reto central que enfrenta el grupo pertinente que toma decisiones es reconciliar una multitud de expresiones e imaginarios acerca del futuro" (2008: 16).

Estamos ahora listos para seguir este didáctico y autorizado texto. De hecho, aquí empezamos a aprender del mismo cuando el autor procede a aclarar su posición pragmática para entender la acción colectiva. Somos en efecto sujetos autobiográficos diferentes. Por tanto, el significado de las cosas es distinto según estemos situados. Así, la 
dificultad obvia para una acción conjunta (joint action) es que cada quien hace lo mismo pero con diferente efecto. De tal suerte que tendremos distintas expresiones acerca de "cómo es" y "cuál es" nuestro lugar en el mundo. En este contexto de la acción conjunta, y desde la perspectiva del pragmatismo volitivo (volitional pragmatism) no hay una expresión o estadio singular sobre el que podamos proyectar nuestras imaginaciones independientes. Más bien son tantos los estadios o fases como participantes haya en la comunidad cuya tarea sea indagar sobre una dirección única de acción para el futuro. Sin embargo, las decisiones se toman sin considerar las preferencias sociales. Se argumenta que la decisión se toma a partir de una razón beneficio-costo mayor que la unidad, o no se aplica determinada política pues a valor presente sus beneficios netos son negativos, o se decide compensar a los perdedores. No es de extrañar entonces, como bien dice Bromley (2006: 129), que se denuncien las decisiones públicas como ineficientes, con sesgos electoreros o motivadas por intereses políticos, irracionales y no en el interés público. ¿Quién, entonces, debiera definir lo que una comunidad debiera querer, o cómo debiera evaluarse lo que debiera hacerse? La cuestión concierne a la legitimidad, la justificación, de tales declaraciones. Pero la legitimación tiene dos componentes: la interna que se refiere a la comunidad que toma las decisiones y la segunda a la comunidad a la que se orientan tales decisiones. La primera tiene que ver con la comprobabilidad, veracidad y consistencia que tales declaraciones tienen de acuerdo con estándares compartidos en la práctica disciplinaria de la que emanan, y la segunda con la coherencia y pertinencia determinadas por los estándares de la comunidad a la que presumiblemente informa y que juzga tales acciones o decisiones. En otras palabras, nos precisa Bromley (2006: 130), se trata de los dos principios del pragmatismo volitivo: creencia o convicción justificada y creencia o convicción valiosa. ${ }^{9}$

Para nuestro autor, la comunidad pertinente por cierto -y lo enfatiza (2006: 141)- podría ser el parlamento, una comisión del legislativo, la junta de directores de un corporativo, un grupo de jueces, un jurado, una familia, un ayuntamiento o el concejo de un pueblo. Así que hay múltiples imaginarios creados que se proyectan hacia múltiples

${ }^{9}$ Bromley las define respectivamente (2006: 218) como las deliberaciones acordadas de una comunidad (generalmente profesional y disciplinaria) de individuos constituidos para producir una creencia acerca de algún ámbito específico de nuestra existencia, y aquella creencia que pueda justificarse ante la más amplia audiencia posible de agentes que piensan y razonan. 
fases por aquellos que mantienen muy diferentes expresiones. No es de extrañar, dice, que la acción colectiva sea controversial. Pero ésta forzará a los participantes a ponerse de acuerdo sobre muchos aspectos y posibles efectos del asunto que se trate. Entonces, el reto fundamental de la acción colectiva es que quien toma las decisiones se dé a la tarea de reconciliar una multitud de expresiones e imaginarios acerca del futuro. No se trata, advierte Bromley, de descubrir la expresión "correcta" (the right expression) de ese conjunto de expresiones, ni de descubrir el "verdadero" imaginario que concuerde con esa expresión correcta. La tarea es, y me permito citarlo en inglés (2006: 142): "...to focus on the various reasons for the disparate expressions, and for the disparate imaginings. Progress in such difficult matters is to be found in reasoned debate. Pragmatists put the matter as the asking for and the giving of reasons".

La decisión emergente de este proceso (debate razonado, expresiones confrontadas, imaginarios distintos) tiene una propiedad que es pertinente señalar: la aceptación por aquellos encargados de formular una acción hacia adelante (2006: 143). Y se pregunta si aceptarla significa que se trata de una decisión que nadie quería; de un compromiso. La decisión que se alcanza representa un consenso, responde. Es decir, para todos, o la mayoría de los participantes, significa lo mejor que puede hacerse bajo las presentes circunstancias: una evidencia de una creencia acordada acerca de lo que debe hacerse; un compromiso para la acción hacia adelante que no está en disputa; no necesariamente la verdad sobre el mejor camino pero sí algo que sería bueno hacer [i!] (2006: 143). Con estas cursivas mías mi pregunta va en el sentido de si estamos preparados para esto, y si no es que sólo podría darse en un sistema verdaderamente democrático, o también en algunas comunidades con un desarrollo institucional que sólo en algunos casos se da, como nos reporta Ostrom (2000).

Una creencia acordada requiere -en el marco de una teoría al respecto- trabajar y discutir qué es lo que debería hacerse. No se trata de calcular los costos con lo que tenemos a la mano sino abandonar el postulado de que los fines disponibles están dados, como insistiría Shackle (1961, cit. por Bromley, 2006: 144-145): Escapar de la necesidad... crear fines, imaginarlos, pensarlos... ya que los pensamientos no están dados.

En fin, y para terminar con esta reflexión, el pragmatismo de Bromley sugiere que la identificación de la decisión correcta es algo que ocurre después de que un consenso se alcanza sobre lo que parece ser lo mejor que debe y puede hacerse bajo las circunstancias del con- 
texto en el que se trabaja. ¿La idea de "second best"?, me pregunto, que casi siempre es la única posible. Sin embargo, lo importante de la propuesta de Bromley es que llegar a un consenso tiene que darse sobre los propósitos que tenemos o podemos imaginarnos del futuro por los que las acciones deben tomarse hoy, en el presente.

Bromley se aleja del relativismo absoluto cuando menciona que es aceptable y legítimo pensar que la ciencia y la religión son actividades para adquirir y categorizar creencias aunque, enfatiza, con propósitos notoriamente diferentes.

Mis preguntas son más pragmáticas:

¿Cómo podemos organizar un debate razonado?

¿Cómo podría un grupo identificar los mejores medios y fines? ${ }^{10}$

¿Cómo podremos alcanzar un consenso sobre lo que parece ser lo mejor que podemos hacer?

¿Cómo podemos lograr ese consenso?

Entiendo que Bromley sostiene que los conceptos y las relaciones económicas no proporcionan información valiosa en el proceso de determinar lo que parece ser lo mejor que debe hacerse en respuesta a situaciones problemáticas específicas (2006: 150). ¿Acaso reconoce el lector esta circunstancia en el contexto actual? La política -dice Bromley- no es sino un proceso continuo de redefinición (reconstrucción) de nuevos ámbitos de la acción individual y grupal. Y, por cierto, una vez que tomamos una decisión debemos estar contentos con ella hasta la próxima sorpresa (2006: 151).

Pero las respuestas a estas preguntas pueden encontrarse precisamente en el último capítulo del libro (2006: 212-224). En otros textos relacionados con este enfoque, en el que se distingue -como hace $\mathrm{Si}$ mmel- entre el contenido de la vida social (la guerra, la familia, la educación, la política) y la forma (el conflicto o el logro del consenso), se ha criticado este enfoque señalando que es simplemente un instrumento analítico para imponer orden en un universo caótico y que sólo describe o busca identificar la estructura y las interacciones que constituyen la vida y el obstinado, pertinaz, irreductible orden social existente. Si lo asociamos al interaccionismo simbólico (Joas, 1990: 117), la crítica ha ido en el sentido de que omite o descuida la estructura

${ }^{10}$ En el enfoque estándar hay por lo menos un criterio que acepta internamente la comunidad epistémica: cuando $B$ - $C$ resulta en un valor positivo o bien si $B / C>1$. El problema es que los fines no necesariamente justifican los medios... 
social, el poder y la historia (y la cultura). Me pregunto entonces si un debate razonado y alcanzar un consenso que no sea necesariamente sólo un compromiso para cambiar con el propósito de quedar igual es posible en el momento, en el contexto actual de nuestro país. Y si la historia y nuestra cultura no están condicionando los momentos políticos para obstruir procesos que permitan tomar decisiones ahora con una imaginación del futuro. ¿Tendrán los presentes actores políticos el poder suficiente, omnisciente (Alexander, 2000: 178), para cambiar las instituciones en nuestro país? ¿Es esto posible?, ¿no hay límites?

Después de varios ejemplos (fumar en espacios públicos, las vacas locas, organismos genéticamente modificados) en los que Bromley pone en acción el abduccionismo (capítulo 10), en el capítulo 11 explica desde el pragmatismo, basado en la voluntad, la deforestación de selvas tropicales, que todos los ambientalistas deberían leer con atención. Y se pregunta ¿cuáles son las razones plausibles para que esto ocurra? Un enfoque pragmático rechazaría explicaciones mecánicas y teleológicas (2006: 171), pues la pregunta del pragmático sería ¿qué evento o circunstancia en el futuro es servido por la deforestación? (p. 174) y no ¿por qué sucede lo que está pasando?

En el capítulo 12 trata sobre las relaciones de propiedad y cómo han evolucionado como institución. Me interesa enfocarme especialmente en los capítulos 13 y 14, penúltimo y último. Según Bromley, la demarcación entre la "ciencia económica" y el "arte de la política" es evidente en la literatura que se refiere a las regulaciones ambientales. La noción de fallas de mercado resulta la metáfora dominante en el análisis económico de la política ambiental. El ejemplo clásico es la contaminación como externalidad atribuible a una falla de mercado (Coase, 1960). La lógica es que si no hay voluntad de pagar por parte de los que contaminan, entonces son socialmente óptimos el aire o el agua sucios.

La prueba definitiva para que el gobierno intervenga es el análisis costo-beneficio. Sin embargo, estos problemas ambientales resultan inevitablemente en una demanda para ser resueltos. Los economistas entonces sospecharían que las organizaciones civiles empujan hacia una excesiva regulación y que los que demandan un ambiente limpio buscan ser "gorrones" (free-riders) del proceso político al obtener un beneficio por el que no tendrían que pagar. Un pragmático, por otra parte, pensaría que más bien habría que exigir que el que contamina pague, o bien, que una actividad que contamina, aunque sea tradicional, no debe continuar una vez que se sabe que perjudica a todos. 
El argumento de la eficiencia en el uso de recursos ambientales se considera incorrecto y resulta una visión estática de la regulación ambiental, en la que tanto la tecnología como los productos, los procesos y las necesidades de los clientes son fijos, según Porter y Van der Linde, citados en Bromley (2006: 205). El paradigma de la competitividad, por el contrario, es dinámico y se basa en la innovación. Las empresas competitivas no son las que tienen menores costos, o consiguen más baratos sus insumos, o alcanzan economías de escala, sino aquellas que desarrollan una capacidad para mejorar e innovar continuamente. En este contexto, según dichos autores, los estándares ambientales pueden estimular las innovaciones en aquellas empresas que entienden que éstas les permiten rebasar el costo de cumplir con las normas y las llevan a obtener ventajas absolutas sobre otras firmas que no tienen que sujetarse a ellas en otras regiones o países. En fin, estimular las innovaciones mediante las regulaciones ambientales pudiera elevar la competitividad de las empresas. Tales argumentos, como dice Bromley, no son creíbles para los economistas ambientales que buscan probar que la normatividad ambiental perjudica a las empresas contaminantes. Se rechaza así la hipótesis de Porter y Van der Linde, pues no sigue "la tradición aceptada". Una visión abductista desde el pragmatismo basado en la voluntad permite ver las cosas de manera diferente. Aparecen entonces dos sorpresas:

1) Las regulaciones ambientales estrictas pueden mejorar la competitividad.

2) A pesar de estrictas medidas o normas ambientales, cuyo costo estimado fue de 102000 millones de dólares en 1992, la economía estadounidense se mantuvo como la más competitiva del mundo.

De hecho, es plausible y esperable que las empresas sean conscientes de que la actitud social sobre la contaminación está cambiando. Ven hacia el futuro y formulan imaginarios respecto del conjunto de medidas y decisiones que habrían de enfrentar en el futuro (2006: 210). Entienden que la tecnología es dinámica y endógena. Se dan cuenta de que deben economizar en lo que tendrán que pagar y en las ventajas de ser adaptadoras precoces y que la receta para producir implica tomar en cuenta los residuos (lo que bajo un régimen de costos resulta un residuo, bajo otro puede no serlo), lo que hace que se considere el uso de insumos y la mejor combinación de éstos. Esto 
implica necesariamente investigación, planeación y reingeniería, lo cual posibilita que la empresa reduzca costos, adopte tecnología y obtenga ventajas sobre la que está disponible. De esta manera, las normas estrictas que hay que enfrentar proporcionan una razón suficiente para que las empresas emprendan un diagnóstico operativo de sus tareas (2006: 211).

Ofrecer una guía para una teoría de las instituciones económicas y para el cambio institucional es el propósito del último capítulo. No se trata, dice Bromley, de una teoría normativa sino de describir las fases por las cuales la acción colectiva va a darse -más que un recuento de lo que realmente sucede.

Distingue el autor teoría de modelo. Este último no es más que un motor analítico estructuralmente dependiente cuya pertinencia reside en su validez interna. Mientras que la teoría es una constelación de conceptos y relaciones que ofrece una estructura plausible para pensar y comprender conexiones particulares de interés, es decir, las razones por las que se dan éstas, lo que permite predecir futuros eventos. Pero ¿Cómo sabemos que tenemos una buena teoría? Ésta debe mantenerse ante retos disciplinarios internos acerca de sus conceptos, propiedades relacionales, explicaciones y predicciones y pasar por varias pruebas antes de considerarse un mecanismo empírico para fijar creencias. Debe expresar hipótesis que traten eventos o situaciones particulares; debe explicar casos diferentes de los imaginados al formular hipótesis; debe hacerse más coherente conforme pasa el tiempo, es decir, ser capaz de extender sus hipótesis a nuevos fenómenos sin que sea necesario modificarla (2006: 213).

El enfoque pragmático pone énfasis no en demostrar cómo el mundo sería mejor o más racional si los individuos y grupos aprendieran a decidir, como proponen los enfoques prescriptivos (lo que hay que hacer). Más bien se trata de resolver problemas y preguntarse por y dar razones para... en términos de qué y cómo creemos que esto o aquello pudiera o debiera... Y recuerda que la política pública es acción colectiva que restringe, o bien libera o expande la acción individual; que altera el ámbito de decisión y el campo de acción de los individuos.

El enfoque pragmático pide que los individuos revelen los propósitos y razones que subyacen detrás de sus creencias actuales. Pero no se sorprende de que diferentes sujetos tengan creencias opuestas acerca de la verdad. De hecho, hay muchas verdades pero no todas las creencias son verdad. De esta suerte el pragmático se pregunta quién debe prevalecer pero, al mismo tiempo, si una verdad autorizada cons- 
tituye una creencia valiosa y si a partir de tal creencia el sujeto está dispuesto a actuar. Esto es lo que se llama pragmatismo basado en la voluntad o volitional pragmatism. La acción colectiva, entonces, es animada por la más amplia justificación de una creencia particular y por la más amplia justificación posible para una acción particular para la que esa creencia particular constituye una razón suficiente (2006: 217). Una razón suficiente es, entonces, el conjunto de pensamientos que nos fija creencias que autorizan la acción.

¿Cómo debemos entender el curso escogido? Algo verdadero -sugiere Bromley (2006: 220)- es lo que en este momento parece plausible de creerse. Una creencia autorizada es necesaria pero no suficiente -advierte- sino cuando se constituye en una creencia valiosa o preciada sobre la que estamos preparados a actuar. En otras palabras, dice Bromley: el grupo decisor -quien sea, pero aceptado socialmente, me imagino- ha encontrado finalmente razón suficiente para alterar arreglos institucionales específicos con el interés de modificar resultados particulares -sean económicos o de cualquier naturaleza-. Y está preparado a defender y justificar sus razones frente a aquellos que permanecen escépticos. Es decir, preparado a ofrecer mejores razones, razones plausibles que nos harán actuar. Esto es -como dice Bromley al final de su libro-, todo lo que hay.

\section{Bibliografía}

Alexander, J. (2000), Las teorías sociológicas desde la Segunda Guerra Mundial, Barcelona, Gedisa.

Bromley, D. (2006), Sufficient Reason. Volitional Pragmatism and the Meaning of Economic Institutions, Princeton, Princeton University Press.

Bromley, D. (2008), "Sufficient Reason: Volitional Pragmatism and the Purposes of the Future", Thinking of the Present by Considering the Future: Lectures on Economics, The Environment and Public Policy, México, Facultad de Economía, UNAM, 24 al 26 de septiembre.

Coase, R. (1960), "The Problem of Social Cost", Journal of Law and Economics, vol. 3, núm. 1, pp. 1-23.

Cortés, F. (2008), "Algunos aspectos de la controversia entre la investigación cualitativa y la investigación cuantitativa”, en F. Cortés, A. Escobar y M. González de la Rocha, Método científico y política social. A propósito de las evaluaciones cualitativas de programas sociales, México, El Colegio de México, pp. 27-58.

Graizbord, B. (2008), “Ambiente y ciencias sociales”, en C. Cadena (comp.), 
Memorias del Coloquio Internacional XX Años de Ciencias Sociales, Zinacantepec, México, El Colegio Mexiquense, pp. 111-138.

Joas, H. (1990), "Interaccionismo simbólico", en A. Giddens et al., La teoría social hoy, Madrid, Alianza, pp. 112-154.

Maffesoli, M. (2008), "La sociología como conocimiento de la socialidad", en C. Cadena (comp.), Memorias del Coloquio Internacional XX Años de Ciencias Sociales, Zinacantepec, México, El Colegio Mexiquense, pp. 71-80.

Ostrom, E. (2000), El gobierno de los bienes comunes. La evolución de las instituciones de acción colectiva, México, Universidad Nacional Autónoma de México/ Fondo de Cultura Económica.

Roemer, J.E. (2008), "The Ethics of Distribution in a Warming Planet”, ponencia presentada en el seminario organizado por el Centro de Estudios Económicos, El Colegio de México, 15 de diciembre (mimeo.). 